\title{
Verbal response-effect compatibility
}

\author{
IRING KOCH \\ Max Planck Institute for Psychological Research, Munich, Germany \\ and \\ WILFRIED KUNDE \\ University of Würzburg, Würzburg, Germany
}

\begin{abstract}
Ideomotor theory states that motor responses are activated by an anticipation of their sensory effects. We assumed that anticipated effects would produce response-effect (R-E) compatibility when there is dimensional overlap of effects and responses. In a four-choice task, visual digit stimuli called for verbal responses (color names). Each response produced a written response-effect on the screen. In different groups, the response-effect was a colored color word (e.g., blue in blue), a white color word, or a colored nonword (Xs in blue). In different blocks, the predictable effects were either incompatible (e.g., response "blue" $\rightarrow$ effect: green) or compatible with the response. We found faster responses with compatible than with incompatible R-E mappings. The compatibility effect was strongest with colored words, intermediate with white words, and smallest with colored nonwords. We conclude that effect anticipation influences response selection on both a perceptual level (related to the word's color) and a conceptual level (related to the word's meaning).
\end{abstract}

Usually, the reason for one's executing an action is to produce an effect on the environment. For example, we press a switch to turn a light on. According to the ideomotor theory of action control, the intended effect can also be the cause of the action. The ideomotor theory can be traced back to an old idea (Harleß, 1861; James, 1890; Lotze, 1852) that has been revived in recent decades (e.g., Greenwald, 1970b; Knuf, Aschersleben, \& Prinz, 2001). Basically, this theory assumes that voluntary action is initiated by activating an anticipatory "image" of the action's intended effects. Returning to our example, the idea of illuminating a room is associated with a certain finger action, so that thinking of the light primes the action of pressing a light switch. This effect anticipation requires reliable learned associations between movements and their sensory effects, which then can function "backward" (i.e., bidirectionally), too, so that activating the representation of the effect activates the associated motor pattern (for reviews, see, e.g., Hommel, 1997; Prinz, 1987, 1997).

The ideomotor theory elegantly solves the problem of how actors intentionally activate specific, complex motor patterns needed to reach their action goal. Still, convinc-

\footnotetext{
The authors thank Peter Graf, Wolfgang Prinz, Robert W. Proctor, and Gregory Stevens for helpful comments on earlier versions of this article, and Renate Tschakert for programming and running the experiments, as well as Heide John for stylistic suggestions. Correspondence should be addressed to I. Koch, Max Planck Institute for Psychological Research, Amalienstrasse 33, D-80799 München, Germany, or to W. Kunde, Department of Psychology III, University of Würzburg, Röntgenring 11, D-97070 Würzburg, Germany (e-mail: iring.koch@ mpipf-muenchen.mpg.de or kunde@psychologie.uni-wuerzburg.de).
}

ing empirical evidence in support of the theory is scarce. As Greenwald (1970b) has suggested, one empirical approach to testing the ideomotor idea is to present previously learned response effects in a transfer phase as stimuli, or together with stimuli. If response-effect (R-E) associations are indeed bidirectional, the effects should prime the associated responses (see Greenwald, 1970a). In fact, Elsner and Hommel (2001; Hommel, 1996) recently demonstrated this priming effect. In an initial training phase, participants performed keypresses that produced simple auditory effects (tones of varying pitch). In a subsequent transfer phase, these effects served as imperative stimuli in a choice-reaction task. As a result, a response was selected more quickly when called by its former effect tone than when triggered by the effect tone of an alternative response.

Such action-priming effects caused by presenting response effects as stimuli demonstrate that R-E learning occurs, which is an important step in exploring the role of response effects in action control (see also Ziessler, 1998). Still, this priming effect does not prove that anticipated action effects ultimately guide action control because these effects were physically presented in the Elsner and Hommel (2001) study, so that effect codes were exogenously activated. In contrast, the original ideomotor idea is that it is the endogenous activation of representations of future, intended effects that actually primes actions.

A recent study by Kunde (2001) sought to assess whether this endogenous effect anticipation indeed occurs. It was reasoned that if action effects really act as mental cues, or internally set stimuli, influences similar to those typically resulting from stimulus perception should result from stimulus (i.e., effect) anticipation as well when 
there is "dimensional overlap" between responses and their effects. In particular, it was predicted that anticipated effects should tend to automatically prime corresponding responses, and hence exert influences of $\mathrm{R}-\mathrm{E}$ compatibility in a similar way as stimuli do in stimulusresponse (S-R) compatibility (see, e.g., Kornblum, Hasbroucq, \& Osman, 1990; Kornblum \& Lee, 1995; Proctor \& Wang, 1997). Observing such R-E compatibility effects would thus suggest that anticipatory effect representations are created and influence response selection, as proposed by the ideomotor theory.

Indeed, Kunde (2001) demonstrated R-E compatibility effects in three experiments. In his Experiment 1, participants responded to one of four centrally presented color stimuli by pressing one of four horizontally aligned response keys. The keypress lighted either a spatially compatible box on the screen (e.g., leftmost key $\rightarrow$ leftmost box) or an incompatible box (e.g., leftmost key $\rightarrow$ rightmost box). R-E mapping was fixed within blocks of trials, so that participants could reliably predict the spatial response-effect within each block. However, the mapping varied between blocks. The results indicated shorter RTs with compatible compared with incompatible R-E mapping. In a second experiment, this finding was generalized from the spatial to the intensity dimension. Here, an R-E compatibility effect was found, in that RTs were shorter when a button pressed strongly (or softly) produced a loud (or soft) tone compared with a mapping in which intensity of response and its effect did not match. In his Experiment 3, a go-no go procedure combined with a free-choice task (cf. Elsner \& Hommel, 2001) was used to exclude any potential influence of stimulus-effect (S-E) or S-R associations that might have occurred in the previous experiments. Again, response initiation was faster with the compatible R-E mapping. Together, these experiments provide clear evidence for R-E compatibility, which, in turn, may be taken as empirical support for the ideomotor principle.

In light of the findings of Kunde (2001), we assume that the explanatory principles for S-R compatibility should also hold for R-E compatibility. If so, it is important to know whether the induced response activation occurs only when responses and effects overlap on a physically defined dimension, like in Kunde's (2001) study, or whether R-E compatibility also generalizes to overlap on more abstract, "conceptual" dimensions. This distinction between physical and conceptual overlap is relevant because anticipated physical effect features can be directly used as motor parameters, if required response features and anticipated effect features match on a physical dimension (e.g., spatiality or intensity). In contrast to overlap on physical dimensions, conceptual overlap (like semantic relations) cannot be directly used to guide action because this overlap exists on a more abstract, motor-unrelated level. There is no doubt, however, that goal-oriented action is often aimed at producing effects that are defined less perceptually than conceptually. That is, such effects depend less on their superficial physical properties than on their abstract meaning. For example, in social interaction, we may want to create a warm interpersonal atmosphere-a situation that cannot easily be described by some primitive sensory features. Therefore, anticipatory effect codes should have the power to influence actions and thus to yield R-E compatibility effects, even when the relation between responses and effects is at a conceptual level rather than at the level of physical features, as was the case in Kunde's (2001) experiments.

The aim of the present study was, thus, to investigate whether R-E compatibility also extends to the conceptual domain. We conducted two experiments in which participants responded to a digit $(1,2,3$, or 4$)$ by saying an arbitrarily mapped color word (blue, yellow, green, or pink). Speech onset produced an immediate visual effect on the screen. In Experiment 1, this effect was either a color word in the congruent color (i.e., the word green in green color) or a colored stimulus without semantic meaning (e.g., four green Xs; in German the color words had four letters). Responses and effects were mapped either compatibly or incompatibly. Compatibility was varied between blocks of trials and effect type (i.e., words vs. Xs) between subjects. For example, in the word group, in a compatible trial, the correct response "blue" to the digit " 3 " would be followed by BLUE in blue, whereas it would be followed by GREEN in green in an incompatible trial. In the color-only group, in a compatible trial, the response "blue" would be followed by XXXX in blue, whereas it would be followed by XXXX in green in an incompatible trial. In Experiment 2, this procedure was replicated by using neutral-color color words (e.g., BLUE in white) as response-effects.

It is important to note that any compatibility effect obtained must be due to the R-E relation because the digits serving as reaction stimuli bore a compatibility relation neither to the responses nor to the effects. Also, this influence must originate from anticipatory effect codes because effects were only presented after the verbal response had been registered. Finally, it is worth noting that the present experimental situation resembles, in a sense, a Stroop (Stroop, 1935) interference situation (see MacLeod, 1991, for a review). That is, the anticipated response effect provides information (word meaning or physical color) that either interferes with or facilitates response (i.e., word pronunciation) selection. However, the crucial difference between the present paradigm and a "standard" Stroop paradigm is that in a Stroop task, the word response (and also the interference) is itself triggered by a color word (or a patch of color), so that the Stroop effect does not relate to effect anticipation. In contrast, an arbitrarily assigned digit triggered the word response in the present experiments, and the interference was due to anticipated rather than to actually presented stimuli.

\section{EXPERIMENT 1}

In Experiment 1, we compared R-E compatibility effects in two groups. In a word group, the R-E words 
were always printed in their congruent colors, and they could be either compatible or incompatible with the response word. In this group, the effective overlap of responses and effects could in principle either refer to the conceptual level (i.e., word meaning) or to the perceptual level (i.e., physical color). To explore whether physical color exerts an influence of its own, we ran an additional color-only group, in which meaningless letter stimuli (colored Xs) were presented as response-effects. For this group, the physical color information of the effects was kept but the word meaning was discarded. To find equally strong effects in both groups would suggest that conceptual information (word meaning) does not play a role for verbal R-E compatibility. However, if we find a stronger effect for the word group than for the color-only group, this would suggest that conceptual information contributes to verbal R-E compatibility in addition to perceptual information.

\section{Method}

Participants. Thirty-two participants (21 female, 11 male) volunteered and received $10 \mathrm{DM}(\$ 4.50)$. Their mean age was 25.5 years. Sixteen participants were randomly assigned to each of the two experimental groups: word group and color-only. Within each group, 8 participants were randomly assigned to each of the two mapping orders: compatible first and incompatible first.

Stimuli and Apparatus. The digits 1, 2, 3, and 4 served as stimuli. Their height was approximately $1.2 \mathrm{~cm}$. The digit stimuli were centrally presented on a computer screen (15-in.) connected to an IBM-compatible PC. Response registration was accomplished with a microphone attached to a headphone set that triggered a voice key. Errors were recorded on specially devised protocol sheets. Error coding was done off line. Registration of a response onset triggered the response effect on the bottom part of the screen. This was either a color word (i.e., the German words gelb, grün, blau, and pink, meaning yellow, green, blue, and pink) presented in the congruent color, or it was four colored Xs, depending on the experimental group (i.e., word vs. color-only). The four colors were easily distinguishable. All effect stimuli consisted of four lowercase letters and measured approximately $0.9 \times 3.6 \mathrm{~cm}$. Viewing distance was approximately $60 \mathrm{~cm}$. Stimulus presentation and response registration was synchronized with the vertical refresh rate of the screen.

Procedure and Design. Testing took place in a dimly lit, soundproof cabin. The participants were informed of their task (respond to digits by saying color words), the four digit S-R mappings they should memorize, and the visual effect of their response, which was not necessary for responding. They were then familiarized with the headphone set and microphone.

Each trial started with a 1,000-msec blank screen. Then, a fixation cross $(0.6 \mathrm{~cm})$ appeared for $100 \mathrm{msec}$. After a 100 -msec blank, the digit was presented and remained until the vocal response was registered. Response registration immediately triggered the presentation of the visual response effect, which was shown for $500 \mathrm{msec}$. The experiment started with 12 practice trials followed by four blocks of 60 trials. The blocks were initiated by pressing any key.

Each participant was confronted with a compatible and an incompatible R-E mapping. Mapping was constant within Blocks 1 and 2 and within Blocks 3 and 4, but changed between the first and second half of the experiment (i.e., after Block 2). The participants were informed of that change and were told that they should continue to respond to the digits as before. Twelve practice trials were run before starting the third block. In contrast to R-E mapping, S-R mapping remained constant for each participant throughout the ex- periment. The order of the R-E mappings (i.e., compatible first vs. incompatible first) was counterbalanced across participants to control for unspecific practice effects. Both S-R and R-E mappings were randomly chosen for each participant and were counterbalanced across participants.

Within each block of 60 trials, each digit stimulus occurred 15 times. The sequence of stimuli (and thus the response and effect sequence) was random, with two constraints: First, immediate stimulus repetitions were excluded, thus reducing the number of possible stimulus alternatives from four to three. Second, we excluded those stimulus transitions in which the subsequent stimulus required a response that corresponded to the last response-effect, thus further reducing the number of alternatives to two. This second constraint was introduced to avoid the response-effect's in trial $n-1$ somehow priming a corresponding response in trial $n$ in the incompatible R-E mapping condition. This constraint was also imposed on the stimulus sequence in the compatible R-E mapping condition although here the exclusion of immediate stimulus repetitions already prevented E-R priming.

\section{Results and Discussion}

For data analysis, the first three trials of each block were discarded as warm-up trials, and errors were excluded from the reaction time (RT) analysis. Error rates were too low $(<0.8 \%$ for both groups) for a meaningful statistical analysis. For RT analysis, RTs above 1,500 msec and below $100 \mathrm{msec}$ were discarded (together, less than $1.7 \%$ in both groups). For the remaining RTs, the mean was calculated as a function of participant and R-E mapping.

When analyzing the R-E compatibility effect, one might suspect that the participants were simply "surprised" when they encountered an incompatible effect and that this surprise could have led to a general slowing down of the responses. To exclude this possibly less interesting explanation, we analyzed RT in Blocks 2 and 4 only - that is, when the participants had had 60 trials of practice with the R-E mappings in addition to the 12 practice trials before starting Block 3 (see Figure 1).

In the word group, RTs with compatible R-E mappings were considerably shorter than with incompatible mapping (632 vs. $712 \mathrm{msec}$ ), indicating an R-E compatibility effect of $80 \mathrm{msec}$. In the color-only group, RTs in compatible trials were also shorter than in incompatible trials ( 669 vs. $684 \mathrm{msec}$ ), but the resulting compatibility effect of $15 \mathrm{msec}$ was much smaller than in the word group. To test this, we ran an analysis of variance (ANOVA) with RT as the dependent variable, R-E compatibility (compatible vs. incompatible) as the independent withinsubjects variable, and experimental group (words vs. color only) as the between-subjects variable. We also included R-E mapping order as the between-subjects variable in order to control for unspecific practice effects (compatible mapping first vs. incompatible mapping first). We obtained a significant compatibility effect $[F(1,28)=$ $\left.17.77, M S_{\mathrm{e}}=2,048, p<.001\right]$ and an interaction of compatibility and experimental group $\left[F(1,28)=8.13, M S_{\mathrm{e}}=\right.$ $2,048, p<.01]$. This interaction shows that the compatibility effect was larger in the word than in the color-only group. We then tested both groups separately. The compatibility effect was significant in the word group $[F(1,14)=$ 


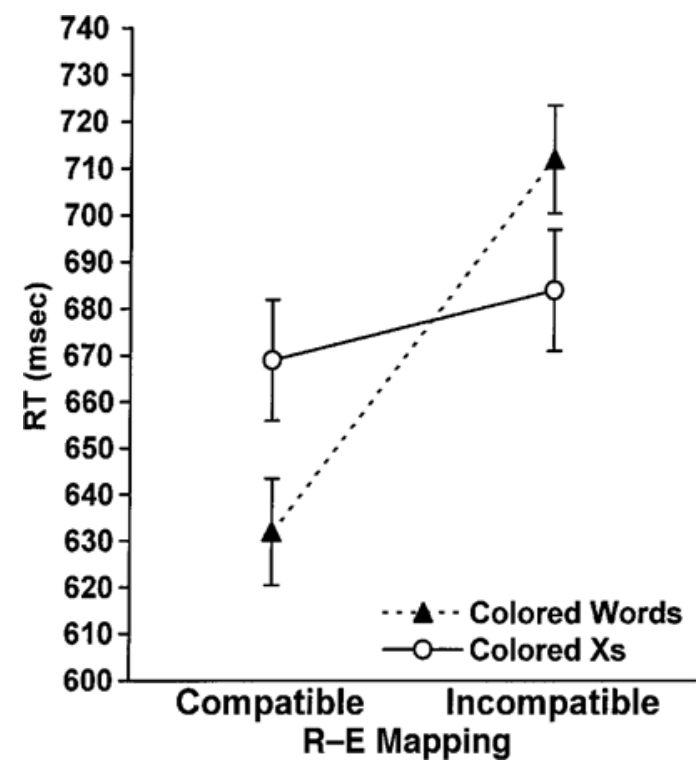

Figure 1. Experiment 1: Mean RT as a function of R-E compatibility and effect type (colored words vs. colored Xs). Bars represent standard errors.

$\left.28.88, M S_{\mathrm{e}}=1,770, p<.001\right]$, but not in the color-only group $(F<1)$. (When we analyzed the compatibility effect by comparing the average of Blocks 1 and 2 with that of Block 3 and 4, we obtained exactly the same pattern of effects, with compatibility effects of 61 and $15 \mathrm{msec}$ for the word and the color group, respectively, suggesting that "surprise" did not play a role.)

Furthermore, the ANOVA, which again included the experimental group variable, revealed that both the main effects of mapping order and experimental group, as well as the interactions of group and mapping order, and the three-way interaction of group, mapping order, and compatibility were not significant (all $F \mathrm{~s}<1)$. However, the interaction of compatibility and mapping order was significant $\left[F(1,28)=29.43, M S_{\mathrm{e}}=2,048, p<.001\right]$, indicating that the compatibility effect was much stronger in the incompatible-first group than in the compatible-first group. This interaction refers to the relation between $\mathrm{R}-\mathrm{E}$ compatibility and nonspecific practice benefits. We note here that this is not critical for the interpretation of the present $\mathrm{R}-\mathrm{E}$ compatibility effect because mapping order was counterbalanced across participants (see Experiment 2 for further discussion).

To account for the R-E compatibility effect, we assume that the representation of the predictable response effects became activated before (or during) response initiation, because otherwise it would be logically impossible for an effect that occurs after the response to exert an influence on this response. This finding of R-E compatibility lends clear support for the idea that anticipating a response-compatible effect primes the action known to produce this effect. The important aspect of the present study, which extends and generalizes Kunde's (2001) results, is that this R-E compatibility effect does not seem to be confined to overlap on a physical dimension but extends to a more abstract, "conceptual" dimension based on word information. The results of Experiment 1 suggest only a minor role for the physically defined color of the response effects, but we further explore this issue in Experiment 2.

\section{EXPERIMENT 2}

Experiment 2 was conducted with neutral-color words to explore further the role of the physically defined color of the response effects for the present verbal R-E compatibility effect. If the compatibility effect is entirely due to word meaning, the compatibility effect should be exactly the same as that found for the word group in Experiment 1 (i.e., with colored effect words). However, if the physical color attribute of the words contributed to the compatibility effect in the word group in Experiment 1 , the compatibility effect should be smaller with neutral than with colored effect words.

\section{Method}

Participants. Thirty-two new participants (20 female, 12 male) volunteered and received $10 \mathrm{DM}$. Their mean age was 24.4 years. Sixteen participants were randomly assigned to each of the two mapping orders: compatible first and incompatible first.

Stimuli, Apparatus, Procedure, and Design. In this experiment, the effect stimuli were color words presented in a neutral color (i.e., white), so that semantic information was retained but physical color information was dropped.

\section{Results and Discussion}

Errors $(2.8 \%)$ and RTs above $1,500 \mathrm{msec}$ and below $100 \mathrm{msec}$ were discarded (1.9\%). The remaining, valid RTs were analyzed as a function of R-E compatibility and mapping order. The compatibility effect amounted to $41 \mathrm{msec}$ (RTs were 664 and $705 \mathrm{msec}$ for compatible and incompatible trials, respectively). An ANOVA yielded a significant effect for compatibility $[F(1,30)=15.55$, $\left.M S_{\mathrm{e}}=1,749, p<.001\right]$, demonstrating that the word information alone was sufficient to produce R-E compatibility. However, we also note that the effect was smaller than that observed with colored effect words in Experiment 1 . Before we discuss this difference, we first turn to the relation between compatibility and practice (i.e., $\mathrm{R}-\mathrm{E}$ mapping order).

The interaction of compatibility and mapping order was, as in Experiment 1, significant $[F(1,30)=23.77$, $\left.M S_{\mathrm{e}}=1,749, p<.001\right]$. The main effect of mapping order was nonsignificant $(F<1)$. The interaction was due to the fact that RT in the incompatible-first group was shorter with the compatible than with the incompatible R-E mapping (628 vs. $720 \mathrm{msec}$ ), whereas in the compatible-first group, RT for the compatible R-E mapping was actually slightly higher than for the incompatible mapping (699 vs. $689 \mathrm{msec}$ ). To elucidate further 


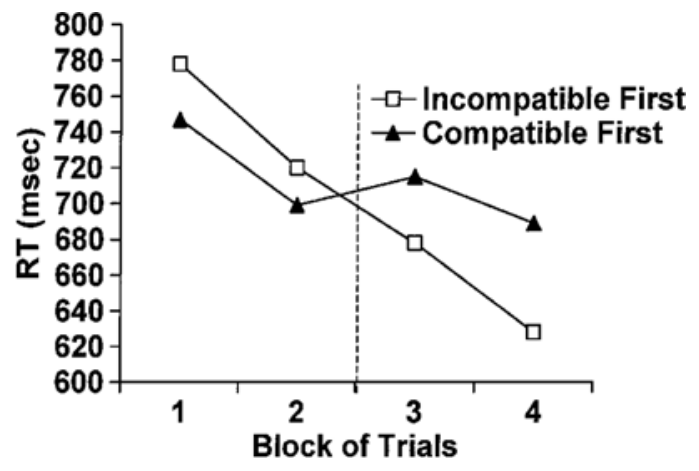

Figure 2. Experiment 2: Mean RT as a function of block of trials and mapping order (incompatible-first group vs. compatiblefirst group).

the relation between compatibility and practice, we analyzed RT as a function of blocks of trials and group (i.e., compatible first vs. incompatible first; see Figure 2).

Figure 2 shows that RT generally decreased as a function of block. This effect is most likely due to increased practice with the $\mathrm{S}-\mathrm{R}$ mappings (which are independent of R-E mappings) and the voice key (i.e., learning to adapt vocalization to the response device). To estimate the compatibility effect, one therefore has to focus on group differences. When looking at the data in this way, the compatibility effect shows up as an interaction between group and practice. In the group that switched from compatible to incompatible R-E mapping (note that $\mathrm{S}-\mathrm{R}$ mapping remained unchanged), the positive practice effect was counteracted by R-E incompatibility (in fact, RT even slightly increased by $17 \mathrm{msec}$ ), whereas RT continued to decrease in the group that switched to the compatible $\mathrm{R}-\mathrm{E}$ mapping. This interaction is most clear in the transition from Block 2 to Block 3. An ANOVA with the variables block and group confirmed the interaction $\left[F(1,30)=8.75, M S_{\mathrm{e}}=1,612, p=.006\right]$. Both the main effect of block $\left[F(1,30)=1.73, M S_{\mathrm{e}}=1,612, p=\right.$ $.199]$ and of group $(F<1)$ were nonsignificant. Note, however, that this ANOVA compares a practiced $\mathrm{R}-\mathrm{E}$ mapping in Block 2 with an unpracticed R-E mapping in Block 3. Therefore, a more appropriate comparison would be between Block 2 and Block 4, and this comparison is logically equivalent to the ANOVA we previously reported on the compatibility effect.

Having elucidated how both practice and R-E compatibility contribute to performance in the present experiments, we now discuss the role of physically defined color information in the response effects. We have argued that the compatibility effect in Experiment 2 should be as large as in the word group of Experiment 1 if the physical color plays no role. However, we observed that the effect was reduced with neutral-color (i.e., white) words as compared with colored words (i.e., 41 vs. $80 \mathrm{msec}$ ). We examined this observation with a betweenexperiments ANOVA with compatibility, mapping order, and experiment as the independent variables and RT (taken from Blocks 2 and 4) as the dependent variable. This analysis yielded significant effects of compatibility $\left[F(1,44)=44.59, M S_{\mathrm{e}}=1,756, p<.001\right]$ and an interaction between compatibility and mapping order $[F(1,44)=$ $\left.42.59, M S_{\mathrm{e}}=1,756, p<.001\right]$. Of importance, the interaction between compatibility and experiment was also significant $\left[F(1,44)=4.554, M S_{\mathrm{e}}=1,756, p<.05\right]$, showing that the compatibility effect was significantly smaller in Experiment 2 than for the word group in Experiment 1 . All other effects were nonsignificant $(F \mathrm{~s}<1)$. In contrast, the 41-msec effect with white words was not significantly larger than the 15-msec effect for the coloronly group in Experiment $1\left[F(1,44)=1.84, M S_{\mathrm{e}}=1,932\right.$, $p=.182]$. Together, the pattern of results suggests that the physically defined color attribute of the response effect significantly contributes to the R-E compatibility effect, although its effect in isolation (without being accompanied by the corresponding word) appears to be rather weak.

\section{GENERAL DISCUSSION}

The aim of the present study was to test whether the R-E compatibility effect observed by Kunde (2001) for overlap on physically defined dimensions of responses and effects also extends to conceptual R-E overlap. To test this, we conducted two experiments with a verbal choice RT task, in which the response (i.e., saying of a color word in response to a digit) triggered a visual effect stimulus. This effect stimulus was compatible to the response in one half of the experiment and incompatible in the other half. In Experiment 1, the type of R-E overlap was varied. A color-only group received colored Xs as response effects, whereas in addition to the physically defined color information, a word group additionally received the conceptual information represented by the meaning of a color word (e.g., blue in blue letters). The results showed that color words led to a stronger R-E compatibility effect than did colored Xs. When we used effect words in neutral color in Experiment 2, the resulting compatibility effect was smaller than with colored words (i.e., the word group in Experiment 1). This shows that the physically defined color attribute is also to some degree retained in the anticipatory effect codes and thus contributes to R-E compatibility.

Together, the results suggest that both the physical color information and the word information contribute as different effect attributes to R-E compatibility. This is an important finding because it extends our knowledge about the range of dimensions on which anticipated response effects can influence action control. We further conclude from our data that the present R-E compatibility effect refers to a more abstract dimension than do those effects recently found on the spatial, intensity, or duration dimensions (Kunde, 2001, in press; Kunde, Koch, $\&$ Hoffmann, in press). Note, however, that the mean effect for colored words was $80 \mathrm{msec}$ but for noncolored words only $41 \mathrm{msec}$, suggesting that the physically de- 
fined color information and the word information both contribute to the effect. However, when tested alone, the color of the Xs had at most a small (and nonsignificant) effect of $15 \mathrm{msec}$. Hence, although the effectiveness of the word information to produce verbal R-E compatibility is clear, further experiments are needed to clarify the role of the color information.

Possibly, the pronounced effect for the group with colored color words resulted from the mutual priming of the physical and verbal attributes of the effect words. Such mutual priming was possible here because the words always appeared in a congruent color. In contrast, mutual priming of physical and verbal effect codes was not possible in the groups with white words or colored Xs because either the physical or verbal attribute was missing. A test of this hypothesis would require nonoverlapping R-E sets (e.g., spoken letters to produce effect color words) when the physical and verbal color information of the effect words are either congruent (e.g., blue in blue) or incongruent (e.g., blue in green). Mutual priming of verbal and physical effect codes would be indicated by superior performance with congruent effect words.

Note that the present finding of a verbal R-E compatibility effect obviously implies that between responses and their effects the contingency was learned, so that the effect could be anticipated. Otherwise it would be logically impossible that future effects would influence present actions. Also, there was no compatibility relation between $\mathrm{S}$ and $\mathrm{R}$ and $\mathrm{S}$ and $\mathrm{E}$. However, one might ask whether $\mathrm{S}-\mathrm{R}$ associations contributed to the observed R-E compatibility effect. Possibly, the compatible effects are just better "reinforcers" for learning arbitrary $\mathrm{S}-\mathrm{R}$ associations, so that $\mathrm{S}-\mathrm{R}$ learning proceeds at a faster pace in compatible than in incompatible conditions. This S-R account, however, is theoretically unsatisfying because it is post hoc and does not specify why compatible effects should be better reinforcers. More importantly, though, the S-R learning hypothesis has been empirically ruled out by other experiments on R-E compatibility. For example, Kunde (2001, Experiment 3) adopted a free-choice procedure in which participants were free to respond to a single go stimulus with either of two responses. Hence, there were no specific reaction stimuli in this procedure and no $\mathrm{S}-\mathrm{R}$ association that could be learned. Nevertheless, strong R-E compatibility effects were observed. Moreover, we have recently found that the compatibility of forthcoming response effects influences responses even when these responses are already fully selected (owing to preceding presentation of a response cue) - that is, when S-R translation had already occurred long before response initiation was required (Kunde et al., in press). This also underlines that $\mathrm{S}-\mathrm{R}$ learning is of minor relevance for $\mathrm{R}-\mathrm{E}$ compatibility effects.

In light of theories of speech production, we could think of two different explanations for our verbal R-E compatibility effect. Current theories assume that the production of a word normally results from the coupling of a conceptual system and an articulatory motor system. In the conceptual system, the correct concept has to be retrieved from the mental lexicon and is then phonologically encoded to allow articulation of the word (see, e.g., Levelt, Roelofs, \& Meyer, 1999, for review). So, as a first explanation, the anticipation of the effect's word meaning could prime retrieval of the lexical concept that corresponds to the required response in the case of $\mathrm{R}-\mathrm{E}$ compatibility. The physically defined color information of the response effect is also related to the lexical concept and should thus prime it as well. A second way that response effects might prime the response is that the anticipated effect stimuli could be recoded into a verbal code. For the color word, this would be structurally equivalent to "reading" an imagined word and for the colored Xs, it would be equivalent to naming the stimulus color. This would then prime the articulatory motor system, because the compatible effect word would prime articulation of the correct response word, whereas the articulatory (or phonological) code for the anticipated incompatible color would prime the incorrect response.

On the basis of the present data, we cannot decide between these two theoretical alternatives. It seems likely to us that phonological priming plays some role (as evidenced, for instance, by nonword priming in pictureword interference; see Levelt et al., 1999), but we believe that conceptual priming (i.e., retrieval from the mental lexicon) also contributes to the verbal R-E compatibility effect.

Note, however, that we derived our prediction of verbal R-E compatibility effects from ideomotor theory of action control. Our results clearly support this theory. A strong version of ideomotor theory would state that anticipated response effects are involved in the actual selection of responses because they automatically prime the corresponding action (see also Hommel, Müsseler, Aschersleben, \& Prinz, 2001). Accordingly, the present R-E compatibility effect would be due to a learning process in which the representation of the effects is "moving" in time ahead of the response to induce automatic priming by conceptual identity with the meaning of the response. This account suggests that effects serve to facilitate response selection and presumes bidirectional priming of response and effect codes (see also Stevens \& Kornblum, 2000).

In comparison, a weaker version of ideomotor theory would assume that R-E compatibility does not facilitate response selection itself but merely the execution of already selected responses. Consider that the activated codes of both the required response and the anticipated response-effect could enter into a joint response representation (see also Aschersleben \& Prinz, 1997). When these two codes are compatible, the joint response representation is more strongly activated (i.e., activation summation) and reaches execution threshold earlier than when both codes are incompatible. Thus, the influence of R-E compatibility might not lie in the priming of re- 
sponse selection but rather in a facilitation of responses that would be specified by an effect-unrelated responseselection process.

Presently, both versions of ideomotor theory are feasible. In fact, in a recent study, we were able to show that response-effects (with $\mathrm{R}-\mathrm{E}$ overlap on the intensity dimension) play a role in both selection and execution of responses (Kunde et al., in press). Hence, it appears that both outlined versions are not mutually exclusive but rather complement each other. Of importance, both speak to the relevance of endogenously activated representations of intended response-effects for the mechanisms of action control. The activation summation account would be a weakened version of ideomotor action control in that anticipated effects come into play only when responses are already selected. This, however, would bring us back to the initial theoretical problem of how actions can be voluntarily selected if they are not controlled by some sort of anticipatory "image" (cf. Greenwald, 1970b) of their intended effects. Therefore, in the absence of contradictory evidence, we prefer the strong version of ideomotor action control.

\section{REFERENCES}

Aschersleben, G., \& Prinz, W. (1997). Delayed auditory feedback in synchronization. Journal of Motor Behavior, 29, 35-46.

Elsner, B., \& Hommel, B. (2001). Effect anticipation and action control. Journal of Experimental Psychology: Human Perception \& Performance, 27, 229-240.

GREenwald, A. G. (1970a). A choice reaction time test for ideomotor theory. Journal of Experimental Psychology, 86, 20-25.

GreenWALD, A. G. (1970b). Sensory feedback mechanisms in performance control: With special reference to the ideo-motor mechanism. Psychological Review, 77, 73-99.

HARLEß, E. (1861). Der Apparat des Willens [The apparatus of the will]. Zeitschrift für Philosophie und philosophische Kritik, 38, 50-73.

Hommel, B. (1996). The cognitive representation of action: Automatic integration of perceived action effects. Psychological Research, 59, 176-186.

Hommel, B. (1997). Toward an action concept model of stimulusresponse compatibility. In B. Hommel \& W. Prinz (Eds.), Theoretical issues in stimulus-response compatibility (pp. 281-320). Amsterdam: Elsevier, North-Holland.

Hommel, B., Müsseler, J., Aschersleben, G., \& Prinz, W. (2001).
The theory of event coding (TEC): A framework for perception and action. Behavioral \& Brain Sciences, 24, 849-937.

JAMES, W. (1890). Principles of psychology. New York: Holt.

Knuf, L., Aschersleben, G., \& Prinz, W. (2001). An analysis of ideomotor action. Journal of Experimental Psychology: General, 130, 779-798.

Kornblum, S., Hasbrouce, T., \& Osman, A. (1990). Dimensional overlap: Cognitive basis for stimulus-response compatibility: A model and taxonomy. Psychological Review, 97, 253-270.

Kornblum, S., \& LeE, J.-W. (1995). Stimulus-response compatibility with relevant and irrelevant stimulus dimensions that do and do not overlap with the response. Journal of Experimental Psychology: Human Perception \& Performance, 21, 855-875.

KUNDE, W. (2001). Response-effect compatibility in manual choice reaction tasks. Journal of Experimental Psychology: Human Perception \& Performance, 27, 387-394.

Kunde, W. (in press). Temporal response-effect compatibility. Psychological Research.

Kunde, W., Koch, I., \& Hoffmann, J. (in press). Anticipated action effects in the selection, initiation, and execution of actions. Quarterly Journal of Experimental Psychology.

Levelt, W. J. M., Roelofs, A., \& Meyer, A. S. (1999). A theory of lexical access in speech production. Behavioral \& Brain Sciences, 22, $1-75$.

Lotze, R. H. (1852). Medicinische Psychologie oder die Physiologie der Seele [Medical psychology or the physiology of the mind]. Leipzig: Weidmann'sche Buchhandlung.

MacLeod, C. M. (1991). Half a century of research on the Stroop effect: An integrative review. Psychological Bulletin, 109, 163-203.

Prinz, W. (1987). Ideo-motor action. In H. Heuer \& A. F. Sanders (Eds.), Perspectives on perception and action (pp. 47-76). Hillsdale, NJ: Erlbaum.

PrINZ, W. (1997). Perception and action planning. European Journal of Cognitive Psychology, 9, 129-154.

Proctor, R. W., \& WANG, H. (1997). Differentiating types of set-level compatibility. In B. Hommel \& W. Prinz (Eds.), Theoretical issues in stimulus-response compatibility (pp. 11-37). Amsterdam: Elsevier, North-Holland.

Stevens, G. T., \& Konnblum, S. (2000, July). Goals and dimensional overlap: The effect of irrelevant response dimensions. Paper presented at the meeting of Attention and Performance XIX, Kloster Irsee.

STROOP, J. R. (1935). Studies of interference in serial verbal reactions. Journal of Experimental Psychology, 18, 643-662.

ZiESSLER, M. (1998). Response-effect learning as a major component of implicit serial learning. Journal of Experimental Psychology: Learning, Memory, \& Cognition, 24, 962-978.

(Manuscript received December 19, 2000 ; revision accepted for publication July 15,2002 .) 